\title{
Power Minimization for Delivering Integrated Multimedia Services over Digital Subscriber Line
}

\author{
Haitao Zheng, Member, IEEE, and K. J. Ray Liu, Senior Member, IEEE
}

\begin{abstract}
We study the problem of designing an efficient resource allocation scheme in the application of providing integrated multimedia services over digital subscriber line. For a predefined quality of service $(\mathrm{QoS})$ requirement and data throughput, we show that the transmitted power consumption can be reduced by applying the parallel transmission framework previously proposed. Data streams are recognized as a set of layers with different data rate and bit error rate requirements. The characteristics of the telephone channel can be utilized to provide unequal error protection naturally and thus efficiently. Simulation results provide comparison of the proposed parallel transmission framework to the existing schemes designed for general data, and demonstrate significant performance improvement, such as 0.5-2 dB power gain.
\end{abstract}

Index Terms - Digital subscriber line, image coding, modulation, multimedia communication, quality of service, resource management, unequal error protection.

\section{INTRODUCTION}

A SYMMETRIC digital subscriber line (ADSL) [1] uses the existing twisted-pair copper loops to offer an effective alternative to the next generation of broadband access networks, and to support high speed communications at very affordable princes. It was specifically designed to exploit the one-way nature of most multimedia communication in which large amounts of information flow toward the user and only a small amount of interactive control information is returned. Typical applications of ADSL are fast access to Internet facilities (e.g., Web services) and fast access to multimedia (e.g., VoD, Digital TV). However, the wide variation in telephone line channel leads to increased difficulty and complexity for any single-carrier system. Multicarrier modulation (MCM) [2], also referred to as orthogonal frequency division multiplexing (OFDM) or discrete multiton (DMT), is currently considered as a standard channel coding scheme for ADSL. Observing that the channel gain and noise vary greatly among the subchannels, a crucial aspect in the design of MCM system is the need to optimize the system transmission bandwidth and power through a loading algorithm [1], [4], [6].

Manuscript received May 5, 1999. This work was supported in part by the NSF NYI Award MIP9457397. Part of this paper was presented at the 1999 IEEE 3rd Workshop on Multimedia Signal Processing, Denmark, September 13-15, titled "Efficient multimedia delivery over digital subscriber line."

$\mathrm{H}$. Zheng was with the Electrical and Computer Engineering Department, University of Maryland, College Park, MD 20742 USA. She is now with Bell-labs, Lucent Technologies, Holmdel, NJ 07733 USA (e-mail: haitaoz@lucent.com).

K. J. R. Liu is with the Department of Electrical and Computer Engineering, and the Institute for Systems Research, University of Maryland, College Park, MD 20742 USA (e-mail: kjrliu @isr.umd.edu).

Publisher Item Identifier S 0733-8716(00)03943-3.
ADSL can support a wide variety of high bandwidth applications that were either not possible or ineffective through the conventional dial-up technologies. Most of those services involve multimedia data, which have quite distinct features compared to ordinary data. Normal data communication requires bit error rate to be maintained at a very low number, while for image and video only certain information requires low bit error rate. Some portion of the content can even sustain high bit error rate without causing too much visible fidelity problem. Therefore, multimedia data are often decomposed into hierarchical perceptually relevant layers, each of which has different perceptual importance and thus different error performance requirement. Under such circumstances, unequal error protection (UEP) outperforms equal error protection. In [9], we show that the proposed parallel transmission can provide proper UEP to the layers, and achieve significant performance improvement compared to the transmission schemes designed primarily for data communication. The goal of the algorithm is to minimize the channel induced distortion, under a given power and bit rate constraint. Therefore, the error performance is decided by the layer's importance and the available channel resources during the optimization.

Some applications allow customers to manipulate the source layers. For example, they are given the freedom to choose the quality associated with each layer. In this case, a layer's importance should not be represented as the average distortion from a single bit error as defined in [9], but according to the customers' request. In particular, the layer's BER performance can be specified by the customers, hence, the objective of the optimization becomes to the satisfy customer's request while using the minimum amount of channel resources. Such consideration would help to increase the number of users that the system can support.

We consider the problem of delivering multistream multimedia data over ADSL, with customer defined BER performance. The layers are not limited to a single source but can be integrated services, such as video, audio, and general data, or different source objects. We develop a loading algorithm which handles the power and rate allocation for a specified BER distribution based on the parallel transmission framework proposed in [9]. The proposed algorithm intends to minimize the overall transmitted power under a given bit rate constraint and QoS requirement. We show that the parallel transmission still outperforms the serial transmission [4]-[7], for this type of application.

This paper is organized as follows. Section II introduces the integrated services, and Section III briefly describes the structure of the parallel transmission framework proposed in [9]. In Section IV, a loading algorithm is developed for a given QoS 
TABLE I

DATA RATE AND BER FOR VARIOUS SERVICES

\begin{tabular}{c|c|c}
\hline Service & Data Rate & BER \\
\hline \hline Video Conferencing & $200 \mathrm{~K}$ bits $/ \mathrm{s}$ & $10^{-6}$ \\
\hline Voice & $10 \mathrm{~K}$ bits $/ \mathrm{s}$ & $10^{-3}$ \\
\hline E-mail & $04 \mathrm{~K}$ bits $/ \mathrm{s}$ & $10^{-5}$ \\
\hline
\end{tabular}

requirement. The proposed loading algorithm is then applied to subband coded image transmission and integrated services transmission over ADSL in Section V. We conclude the paper in Section VI.

\section{INTEGRATED SERVICES}

The popularity of the Internet is fueling the emergence of multimedia applications. A mixture of data, voice, and video can provide entertainment, online services, interactive shopping, and video conferencing. Therefore, modern wireline communication should provide an integration of the traffic flows from different services. Those services are associated with different data rate and different QoS requirement (in terms of BER). A typical example is shown in Table I, which involves video conferencing, voice data, and e-mail. Those services can be viewed as source layers with different bit rates and BER requirements. We assume that each service is accompanied by an efficient rate control scheme to achieve constant bit rate.

In addition to integrated services, single source stream can also be decomposed into several substreams. Layer or scalable coded multimedia data, such as subband coded image and video, are typical examples. Each layer may be associated with different QoS requirement. MPEG4 is an object based multimedia coding standard [8]. It represents the source data in terms of "audio/visual objects," or AVO's, organized in a hierarchical fashion. Examples include background, picture of a talking person, and voice of the speaker, etc. Such a coding scheme has the advantage of allowing extraction and editing of an object, or having an object available in a scalable form. The background and speaker are coded as different objects. Some customers want to observe the speaker clearly and can tolerate some effects in the background and the voice, while the others are more interested in background. Upon this, the system should allow the customers to define the QoS of each object. Therefore, the objects are equivalent to the layers with different QoS.

\section{Parallel TRansmission Framework}

As shown in [9], telephone channels are mostly spectrally shaped, where the channel gain and noise variance differ tremendously among the subchannels. The loading algorithms developed for data transmission deemed this property as a disadvantage and tried to eliminate it via a loading algorithm. These algorithms were shown in [9] as serial transmission. In contrast, we utilized it as an advantage and proposed a parallel transmission framework which transmits the layers simultaneously, each occupying a set of subchannels [9], [10]. Fig. 1 shows the system structure.

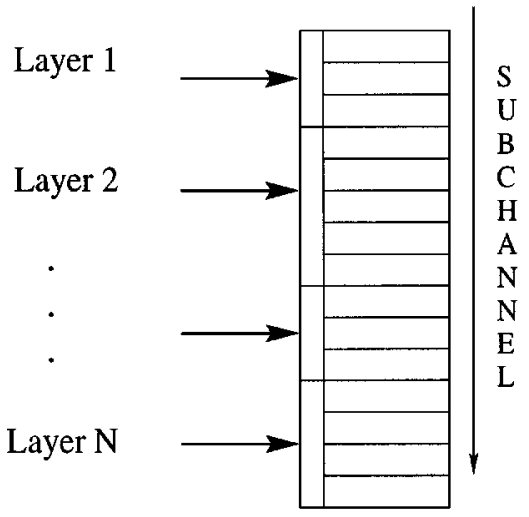

(a)

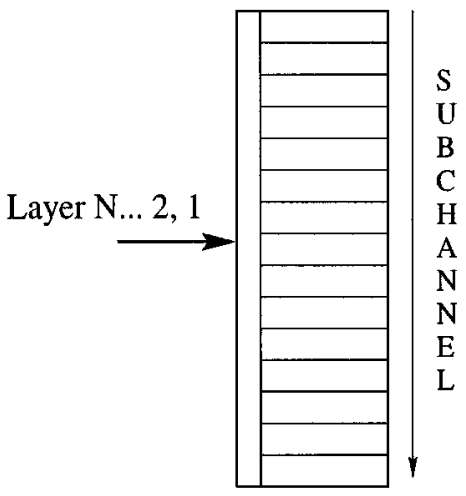

(b)

Fig. 1. The structure of the (a) parallel and (b) serial transmission systems.

The goal in [9] is to minimize the channel induced distortion. Therefore, the layer's importance is defined as the average distortion caused by a single bit error, or the weighting factor. Larger weighting factor leads to higher importance. Therefore, QoS is not customer defined; and it depends on the source characteristics. The layers with higher importance are transmitted through the subchannels with higher channel gain to noise ratio (CGNR). Such assignment can provide UEP naturally even with equal power and bit rate assignment among the subchannels. To match the UEP to the importance distribution, a loading algorithm was developed to optimize the power and bit rate allocation. Since the serial transmission transmits the layers consecutively, the performance bottleneck is the huge power consumption for the important layers which requires very low error rate even at the subchannels with low CGNR. The advantage of the parallel transmission is by transmitting the layers requiring low error rate through only the subchannels with high CGNR, the corresponding power consumption can be reduced at the cost of the power consumption increase at the layers with high error rate. The loading algorithm intends to find the best subchannel to layer assignment as to achieve the optimal error rate distribution and thus the minimum channel distortion.

For integrated services with a mixture of multiple services, the serial transmission needs to compute the subchannel power and bit rate assignment as well as the total power assigned to individual services, which involves frequent change at both the transmitter and the receiver. Rather than setting up different transmission parameters to carry multiple services, the parallel 
transmission can integrate various traffic flows with different service requirements based on the current channel characteristics, and at the same time reduce the usage of transmitted power as we will show in later sections. Since the transmission parameters hold the same during the transmission, the parallel transmission achieves significant complexity savings at transmitter/receivers.

\section{THE POWER OPTIMIZATION FOR GIVEN BER's}

This section aims to develop a loading algorithm that achieves a specified BER distribution by distributing the transmitted power and bit rate among the subchannels. We assume that the layers are ordered in increasing BER order, and the subchannels are indexed in decreasing CGNR order. Similar to the loading algorithm in [9], the optimization can be divided into the following two steps.

1) The Optimization Inside a Layer: Since the transmission time of the layers are fixed to be the same, for a given data throughput $B_{T}$, the number of bits of layer $i$ to be transmitted at each ADSL is given by

$$
B_{i}=\frac{B i t_{i} B_{T}}{B i t_{\text {total }}}
$$

where $B i t_{i}$ represents the total bits of layer $i$ and $B i t_{\text {total }}$ represents the total bits of all the layers. Define the number of subchannels transmitting layer $n$ as $C_{i}, i=1 \ldots N$. For a given $\left\{C_{i}\right\}_{i=1}^{N}$, the algorithm computes the channel gain and noise variance of the subchannels transmitting each layer and optimizes the power and bit rate allocation for each layer independently. The independence is also due to the knowledge of BER performance. For layer $i \in=\{1 \ldots N\}$, the loading algorithm for data transmission can be applied to equalize the error performance to achieve $B E R_{i}$ and data throughput $B_{i}$. Fig. 2 shows the subchannel BER performance.

\section{A. Bit and Power Loading for a Given Number of Subchannels}

For each layer, if the number of subchannels occupied is decided, the loading algorithm developed for data communications can be used to allocate the power and bit rate among the subchannels transmitting the same layer, which is described in this section.

Each subchannel is equivalent to an AWGN channel, with input energy $E_{s}$, channel gain to noise ratio $G$. And the maximum data rate or capacity is

$$
C=\log _{2}\left(1+E_{s} G\right) \text {. }
$$

Any reliable and implementable system has to transmit a data rate below such capacity. For a given symbol error probability $P e$ and rate $b$, an SNR gap is defined as

$$
\Gamma=\frac{2^{C}-1}{2^{b}-1}=\frac{\mathrm{SNR}}{2^{b}-1}
$$

where SNR $=E_{s} G$. The gap measures the SNR distance from capacity. For uncoded QAM, $P e=10^{-6}$ corresponds to a con-

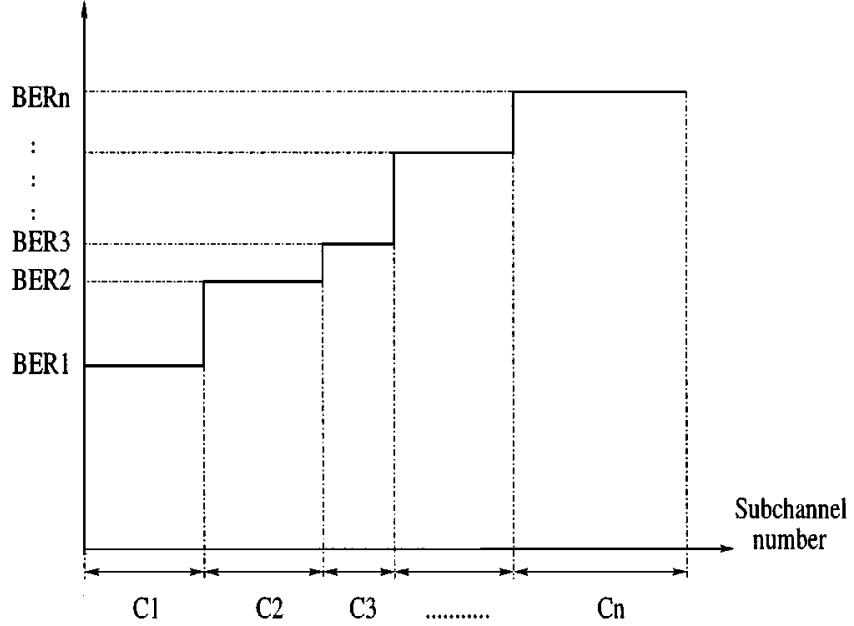

Fig. 2. BER distribution for the subchannels.

stant $\Gamma=8.8 \mathrm{~dB}$. Equivalently, the rate $R$ can be expressed as a function of SNR and $\Gamma$,

$$
b=\log _{2}\left(1+\frac{\mathrm{SNR}}{\Gamma}\right) .
$$

The goal is to minimize the overall transmitted power $E$, for a set of $M$ parallel subchannels with a given throughput and a specific SNR gap $\Gamma$. The throughput is the total bit sum over the subchannels. Water-filling algorithm computes the bit rate using (4), so that the bit rate can be any real number. To avoid this, we use the optimum discrete loading algorithm described in [3]. For subchannel $m$, if using QAM, the transmitted power can be expressed via the gap approximation as

$$
E_{m}\left(b_{m}\right)=\frac{\Gamma}{g_{m}}\left(2^{b_{m}}-1\right)
$$

where $b_{m}$ and $g_{m}$ are the bit rate and CGNR of subchannel $m$, respectively. Therefore, the incremental energy $e_{m}\left(b_{m}\right)$ [3], defined as the amount of additional power required to send the $b_{m}^{\text {th }}$ bit, can be computed as

$$
\begin{aligned}
e_{m}\left(b_{m}\right) & =E_{m}\left(b_{m}\right)-E_{m}\left(b_{m}-1\right) \\
& =\frac{\Gamma}{g_{m}} 2^{b_{m}-1} .
\end{aligned}
$$

A bit distribution $\vec{b}=b_{1}, b_{2}, \ldots, b_{C}$, where $C$ is the number of subchannels, is efficient if no movement of a bit from one subchannel to another can reduce the overall transmitted power, i.e.,

$$
\max _{m \in[1, C]} e_{m}\left(b_{m}\right) \leq \min _{n \in[1, C]} e_{n}\left(b_{n}+1\right) .
$$

In order to make the bit distribution efficient, the loading algorithm starts from an arbitrary $\vec{b}$, and approaches the optimal distribution successively. Each time the algorithm selects a subchannel with the minimum $e_{m}\left(b_{m}+1\right)$, a subchannel with the maximum $e_{n}\left(b_{n}\right)$, and move one bit from $n$ to $m$. It repeats the procedure until the efficiency is achieved.

The loading algorithm can start from any arbitrary $\left\{b_{m}\right\}_{m=1}^{M}$ distributions, and approach the optimal solution successively. The algorithm first makes the distribution $\left\{b_{m}\right\}$ efficient by 
moving the bits among the subchannels. After this, if the $\sum_{m=1}^{M} b_{m}$ is too high, the algorithm finds the subchannel with the most power reduction per bit and reduce the bit rate by 1 . On the other hand, if the sum is lower than $R$, the bit rate of the subchannel with the least power increase per bit is increased. Therefore, the initial selection of distribution is very important. To maximize the overall bit rate under a given power constraint, the same algorithm can be applied. The power constraint corresponds to the frame power, defined as the sum of the transmitted power over all the subchannels. The algorithm first makes the starting distribution efficient and then if the total power consumption does not satisfy the constraint, increase the bit rate of the subchannel with the least power increase per bit if the power sum is sufficiently below the constraint, or decrease the bit rate of the subchannel with the most power reduction if the power sum is above the constraint.

1) The Optimization Among the Layers: This level of optimization decides the distribution of $\left\{C_{i}\right\}_{i=1}^{N}$, with a constraint $\sum_{i=1}^{N} C_{i} \leq C_{T}$, where $C_{T}$ is the number of subchannels in the MCM system. We assume that each subchannel can only transmit a single layer, and therefore $C_{i} \in[1,2, \ldots]$.

\section{B. The Number of Subchannels Allocation}

As described in [9], the layers with higher importance are transmitted through the subchannels with higher CGNR value. We assume that the subchannel indexes are arranged in such a way that the subchannels with larger index have lower CGNR value. The source layers are arranged in decreasing QoS requirement order, e.g., larger index leads to higher error rate requirement. Therefore, if the number of subchannels assigned to each layer is defined, the subchannels assigned to each layer are arranged accordingly.

The number of subchannels occupied by the layers $\left\{C_{i}\right\}_{I=1}^{N}$ is used as optimization parameter. In practical realization, the number of modulator/demodulators at transmitter and receiver is limited to $\left[R_{\min }, R_{\max }\right]$. Using QAM as an example, if $\left[R_{\min }, R_{\max }\right]=[2,6]$, then the allowed modulation types are QAM4, QAM8, QAM16, QAM32, QAM64. The number of subchannels transmitting layer $i, i=1 \cdots N$, is bounded by

$$
\begin{aligned}
C_{i, \min } & =\frac{B_{i}}{R_{\max }} \\
C_{i, \max } & =\frac{B_{i}}{R_{\text {min }}} .
\end{aligned}
$$

In [9], $C_{i}$ is defined by $B_{i} / R_{i}$ where $R_{i}$ represents the average bit rate per subchannel for layer $i$ and is integer valued. As such, the selection of $C_{i}$ is limited. In this paper, we allow $C_{i}$ to be selected from any integer number between $\left[C_{i, \min }, C_{i, \max }\right]$. The number of subchannels used for all the layers is limited to $C_{T}$.

We define $Q_{j, k}$ as the total power variation by increasing the number of subchannels that layer $k$ occupies by one and decreasing the number of subchannels that layer $j$ occupies by one. Or, $Q_{j, k}$ represents the overall power consumption increase for moving one subchannel from layer $j$ to layer $k$. Here the power variation is defined as the difference between the power consumption with and without the subchannel movement. $Q_{j, k}>0$ yields the conclusion that the movement increases the power consumption and thus is not feasible. Since the total subchannels used can be less than the subchannels available $C_{T}$, we introduce an $N+1$ th layer which represents the subchannels not in use. The power consumption and power variation associated with this layer are always zero. We define the subchannel distribution efficiency and total subchannel efficiency.

Definition 1-Subchannel Distribution Efficiency: For a given number of total subchannels in use, if no movement of a single subchannel from one layer to another, excluding layer $N+1$, can reduce the power consumption, then the subchannel distribution efficiency is achieved. Mathematically,

$$
Q_{j, k}>0, \quad j, k \in\left\{[1, N], C_{j}>C_{j, \min }, C_{k}<C_{k, \max }\right\} .
$$

Definition 2-Total Subchannel Efficiency: This is to decide the optimal number of subchannels occupied by all the layers, defined as $\sum_{i=1}^{N} C_{i}=C_{T}-C_{N+1}$. If no movement of a single subchannel from layer $N+1$ to layer $l \in[1, N]$ can reduce the power consumption, then the total subchannel efficiency is achieved. Mathematically,

$$
Q_{N+1, k}>0, \quad k \in\left\{[1, N], C_{k}<C_{k, \max }\right\} .
$$

The loading algorithm starts from the subchannel distribution $\left\{C_{1}, \min , C_{2}, \min , \ldots, C_{N, \min }\right\}$, which satisfies the subchannel distribution efficiency. If this distribution also satisfies the total subchannel efficiency, then the solution is achieved, otherwise the algorithm finds the layer that maximizes the power reduction by adding one subchannel to it. After updating the layers, the subchannel distribution efficiency may not hold and, if so, the subchannel distribution $\left\{C_{i}\right\}_{i=1}^{N}$ is optimized. Overall, a distribution that achieves both the subchannel distribution efficiency and the total subchannel efficiency is deemed as the optimal solution.

The complexity of the algorithm depends on the computation of $Q_{j, k}$. The following theorem is derived to further reduce the complexity. We define the starting subchannel for a particular layer as the subchannel with the lowest index number assigned to this layer, and the ending subchannel as the subchannel with the highest index number.

Theorem 1: Moving subchannels between layer $j$ and layer $k(j>k)$ will only affect the transmitted power and bit rate distribution of the subchannels assigned to layer $l$ where $k \leq$ $l \leq j$.

Proof: Increasing or decreasing one subchannel from layer $k$ will not affect the layers numbered less than $k$ since the ending subchannel of layer $k-1$ remains the same. Therefore, moving subchannels between layer $j$ and $k$ will not affect the transmitted power and bit rate allocation for the layers numbered less than $k$. The index of the starting subchannel of layer $j+1$ will not change since the total number occupied by layer 1 through $j$ remains the same. Therefore, this movement will not change the allocation for the layers numbered larger than $j$.

From Theorem 1, we divide the computation into the following two categories.

1) $j>k$ : As can be seen from Fig. 3 and Theorem 1, the power and bit rate allocation for the layers with index number 

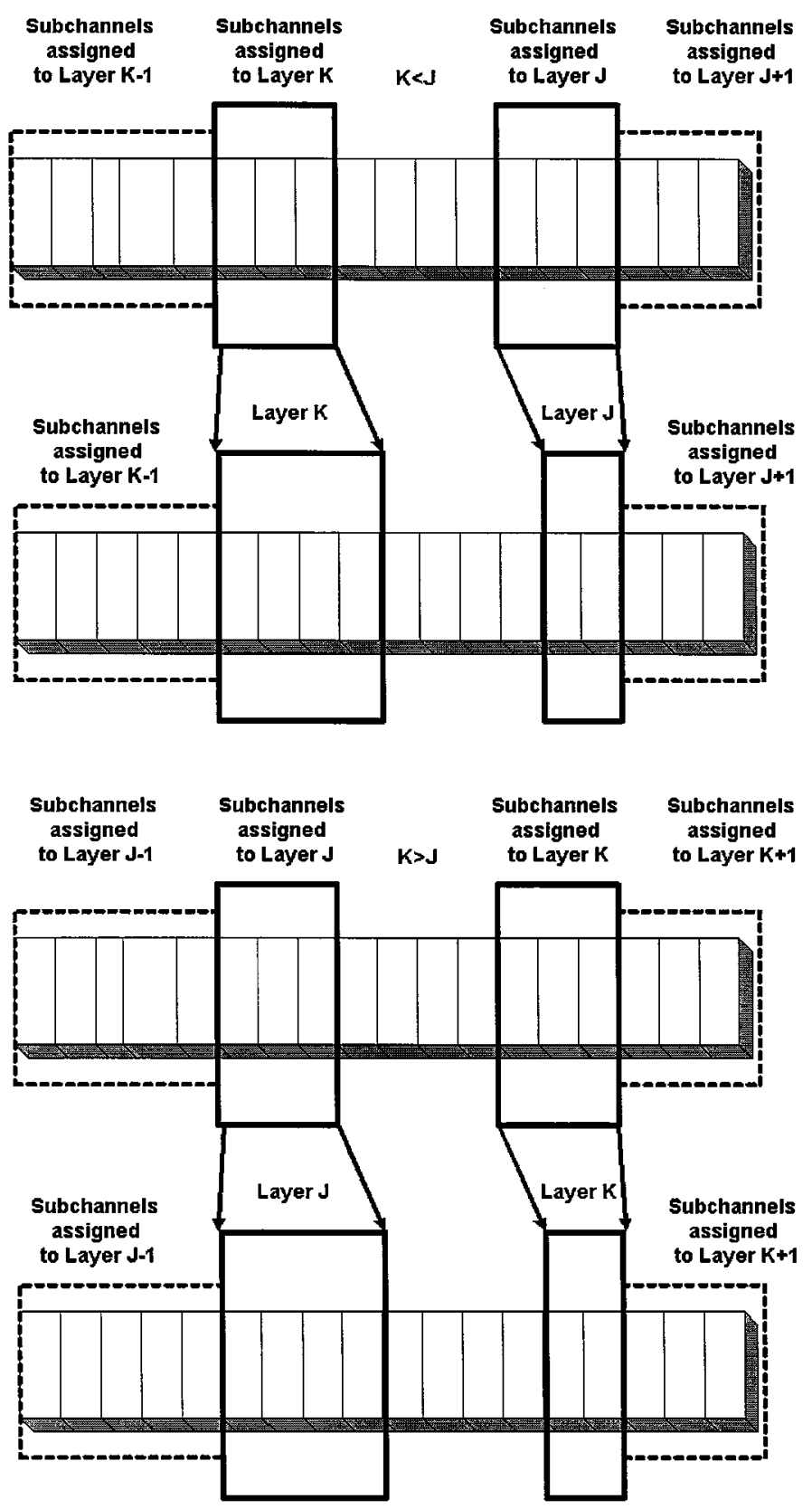

Fig. 3. Effect of subchannel movement.

less than $k$ or larger than $j$ will not be affected. The starting subchannel index for layer $k$ will not change but the indexes for layer $k+1, \ldots, j$ will increase by one. Therefore, the power variation is associated with layers $k, k+1, \ldots, j$; and $Q_{j, k}$ contains the following three factors.

- $p_{l}^{\uparrow}, l \in[k+1, k+2, \ldots, j-1]$ : the power variation at layer $l$ due to the subchannel index increase. Here the number of subchannels occupied by layer $l$ remains unchanged. Since the subchannel CGNR decreases as the index increases, additional transmitted power is required to satisfy the QoS requirement. Therefore, $\hat{p_{l}}>0$.

- $p_{k+}$ : the power variation at layer $k$ because of the additional subchannel. Because of the extra bandwidth, it is straightforward that $p_{k+} \leq 0$.
- $p_{j-}^{\uparrow}$ : the power variation at layer $j$ due to the subchannel loss and the index increase. This is equivalent to removing the starting subchannel of layer $j$ and making the subchannel next to it the new starting subchannel. Therefore, the average subchannel CGNR degrades which leads to higher transmitted power consumption $p_{j-}^{\uparrow}>0$.

Overall

$$
Q_{j, k}=\sum_{l=k+1}^{j-1} p_{l}^{\uparrow}+p_{k+}+p_{j-}^{\uparrow}, \quad j>k .
$$

For layer $N+1, p_{N+1}^{\uparrow}=p_{N+1+}=p_{N+1-}^{\uparrow}=0$. Since the goal is to achieve negative power variation (power reduction), so that if $p_{k+}+p_{j-}^{\uparrow} \geq 0$, then moving subchannel from $j$ to $k$ is not feasible. Furthermore, $Q_{j, k}$ can be calculated recursively, since

$$
Q_{j, k}-Q_{j, k+1}=p_{k+1}^{\uparrow}-p_{k+1,+}+p_{k+} .
$$

Therefore, we start from $Q_{j, j-1}$ since it involves the least number of $p_{l}^{\uparrow}$ computation

$$
\begin{aligned}
Q_{j, j-1} & =p_{j-1,+}+p_{j-}^{\uparrow} \\
Q_{j, k} & =Q_{j, k+1}+p_{k+1}^{\uparrow}-p_{k+1,+}+p_{k+}, \\
k & =j-2, \ldots, 1 .
\end{aligned}
$$

2) $j<k$ : The layers with index number less than $j$ or larger than $k$ will not be affected. The starting subchannel index of layer $j+1, \ldots, k$ will decrease by one. Similarly, the power variation has three contributions.

- $p_{l}^{\downarrow}, l \in[j+1, j+2, \ldots, k-1]$ : the power variation at layer $l$ caused by the subchannel index decrease without changing the number of subchannels. Since the subchannel CGNR increases as the index number decreases, the performance is improved and this value is always nonpositive.

- $p_{k+}^{\downarrow}$ : the power variation at layer $k$ for an additional subchannel as the starting subchannel. It is equivalent to adding more bandwidth to layer $k$ and $p_{k+}^{\downarrow} \leq 0$.

- $p_{j-}$ : The power variation at layer $j$ by removing one subchannel. It is obvious that $p_{j-} \geq 0$.

The above yields

$$
Q_{j, k}=\sum_{l=j+1}^{k-1} p_{l}^{\downarrow}+p_{k+}^{\downarrow}+p_{j-}, \quad j<k .
$$

Similarly, $Q_{j, k}$ can be computed recursively, starting from $Q_{j, j+1}$

$$
\begin{aligned}
Q_{j, j+1} & =p_{j+1,+}^{\downarrow}+p_{j-} \\
Q_{j, k+1} & =Q_{j, k}+p_{k}^{\downarrow}+p_{k+1,+}^{\downarrow}-p_{k,+}^{\downarrow}
\end{aligned}
$$

From the above analysis, in order to decide whether to move one subchannel from layer to layer, the following parameter set $P S_{l}=\left\{p_{l_{+}}, \hat{p_{l-}}, p_{l}^{\uparrow}, p_{l}^{\downarrow}, p_{l+}^{\downarrow}, p_{l_{-}}\right\}, l=1, \ldots, N+1$ is required. If a subchannel movement from layer $j$ to layer $k$ can generate the maximum power consumption reduction, then only the $P S_{l}, l=[j, \ldots, k]$ if $j<k$ or $l=[k, \ldots, j]$ if $j>k$ requires updating.

Next, we describe the whole algorithm. 
TABLE II

SimUlation Result For SubBand Coded IMAge, $B_{T}=512$

\begin{tabular}{c|c|c|cc|c}
\hline \multicolumn{3}{c|}{ BER } & \multicolumn{2}{|l}{ Average Power(dB) } & \multirow{2}{*}{ Performance } \\
\cline { 1 - 4 } BER1 & BER2 & BER3 & Parallel & Serial & PSNR(dB) \\
\hline \hline $6 \cdot 10^{-6}$ & $7 \cdot 10^{-6}$ & $7 \cdot 10^{-6}$ & 21.38 & 21.61 & 33.30 \\
\hline $10^{-6}$ & $10^{-4}$ & $10^{-3}$ & 18.26 & 19.27 & 32.56 \\
\hline $7 \cdot 10^{-6}$ & $5 \cdot 10^{-4}$ & $10^{-2}$ & 16.44 & 17.85 & 31.06 \\
\hline $3 \cdot 10^{-3}$ & $7 \cdot 10^{-3}$ & $10^{-2}$ & 15.60 & 16.20 & 27.90 \\
\hline
\end{tabular}

\section{Loading Algorithm for Power Minimization}

\section{1) Initialization:}

- Sort the layers in decreasing importance order, sort the subchannels in decreasing CGNR order.

- Compute $\left\{C_{i, \min }, C_{i, \max }\right\}_{i=1}^{N}$ as in (8).

- Set $C_{N+1, \min }=C_{T}-\sum_{i=1}^{N} C_{i, \max }$, and $C_{N+1, \max }=$ $C_{T}-\sum_{i=1}^{N} C_{i, \text { min. }}$.

- Set $t=0$. Set $\left\{C_{i}\right\}_{i=1}^{N}=\left\{C_{i, \min }\right\}_{i=1}^{N}$, and $C_{N+1}=$ $C_{N+1, \max }$. Compute the power and bit rate distribution for layer 1 to $N$ using the algorithm described in Section IV-A. After the allocation, the bit distribution of the subchannels assigned to each individual layer should satisfy the bit distribution efficiency criterion, meaning that no movement of a bit from one subchannel to another within the same layer can further reduce the transmitted power consumption

$$
\begin{aligned}
& \max _{1 \leq p \leq C_{i}}\left\{e_{p}^{i}\left(b_{p}^{i}\right)\right\} \\
& \quad \leq \min _{1 \leq q \leq C_{i}}\left\{e_{q}^{i}\left(b_{q}^{i}+1\right)\right\}, \quad i=1 \ldots N
\end{aligned}
$$

where $e_{p}^{i}\left(b_{p}^{i}\right)$ stands for the addition power required to send the $b_{p}^{t h}$ bit with respect to the $b_{p}-1^{\text {th }}$ bit at subchannel $p$ for layer $i$.

- Set $C=\sum_{i=1}^{N} C_{i}^{t}$, (here assume $C<C_{T}$ ).

- Here the subchannel distribution efficiency is satisfied since the layers are associated with the minimum number of subchannels.

- Compute $P S_{l}=\left\{p_{l+}, \hat{p_{l-}}, \hat{p_{l}^{\uparrow}}, p_{l}^{\downarrow}, p_{l+}^{\downarrow}, p_{l-}\right\}, l=$ $1, \ldots, N$. Here if the subchannel index changes, the bit efficiency needs to be investigated and optimized.

- Set $P S_{N+1}=\{0,0,0,0,0,0\}, t=0$.

2) Achieve Efficiency:

a) $t=t+1$.

b) Test the total subchannel efficiency (note that at this point the subchannel distribution efficiency still holds).

- Compute $Q_{N+1, k}=\sum_{l=k+1}^{N} p_{l}^{\uparrow}+p_{k+}, k=$ $1 \ldots N$.

- Find

$m=\arg \max _{\left\{k \in[1, N], Q_{N+1, k}<0\right\}}-Q_{N+1, k}$.

- If no feasible $m$ is find, then the total subchannel efficiency is achieved and therefore the solution is reached. Algorithm end.

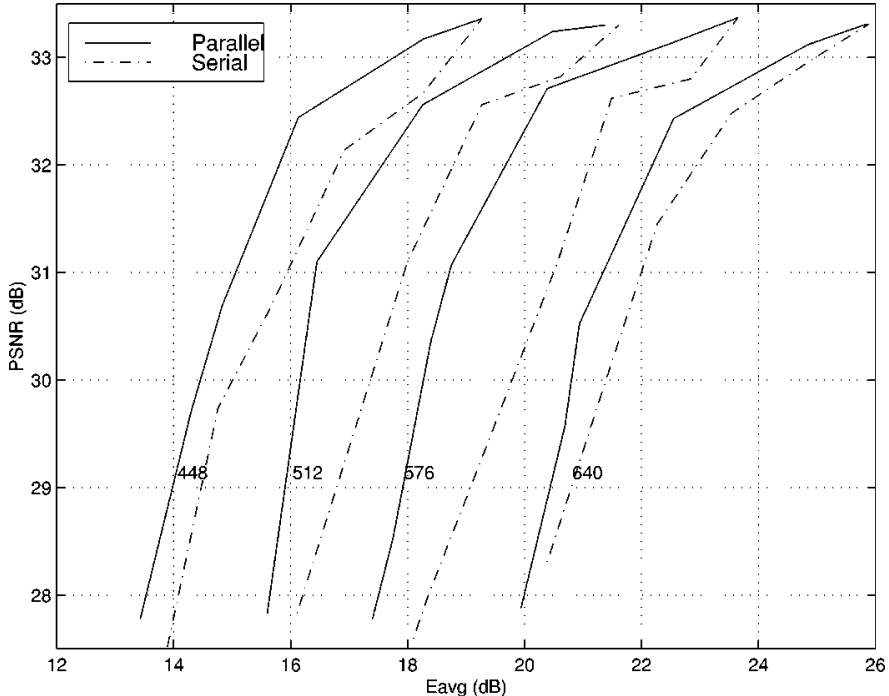

Fig. 4. PSNR versus average power performance comparison at $0.5 \mathrm{bpp}$ source rate.

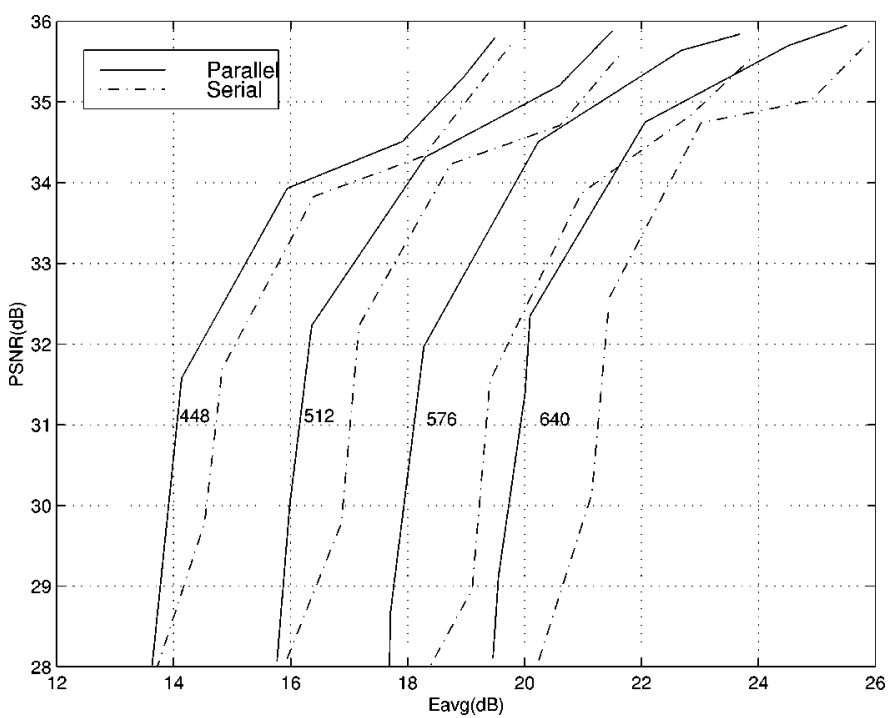

Fig. 5. PSNR versus average power performance comparison at $1.0 \mathrm{bpp}$ source rate.

Otherwise, set $C_{m}=C_{m}+1$ and update the subchannel indexes of layer $m$ through $N$, compute $\left\{P S_{l}\right\}_{l=m}^{N}$.

c) Test the subchannel distribution efficiency.

- For $j=1$ to $N$,

If $C_{j}=C_{j, \min }$ go to b), otherwise

For $k=1$ to $N, k \neq j$

- if $C_{k}<C_{k, \max }$ compute

$Q_{j, k}= \begin{cases}\sum_{l=k+1}^{j-1} p_{l}^{\uparrow}+p_{k+}+p_{j-}^{\uparrow}, & j>k \\ \sum_{l=j+1}^{k-1} p_{l}^{\downarrow}+p_{k+}^{\downarrow}+p_{j-}, & j<k\end{cases}$

- end iteration for $k$

- We can also use (13) and (15) to compute $Q_{j, k}$ recursively. 


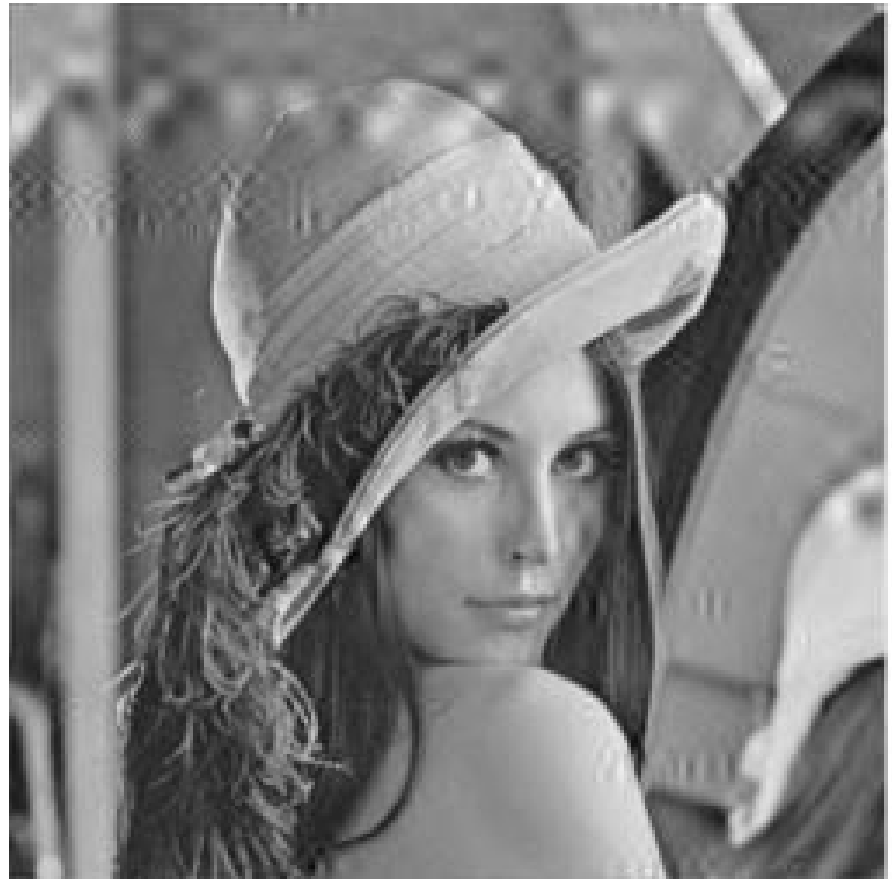

(a)

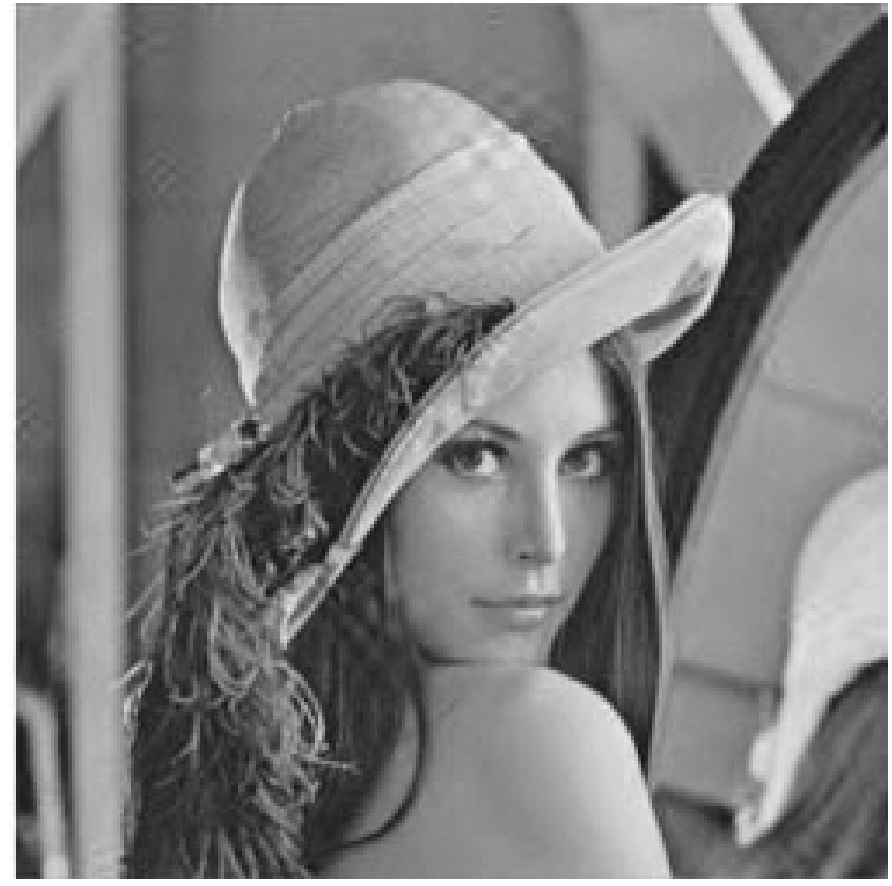

(b)

Fig. 6. Received image for $E_{\mathrm{avg}}=17 \mathrm{~dB}$ and $B_{T}=512$. (a) Serial transmission. (b) Parallel transmission.

- Find $j_{a}=\arg \max \left\{k \in[1, N+1], Q_{j, k}<0, C_{k}<C_{k}, \max \right\}$ $-Q_{j, k}$.

- $q_{j}=-Q_{j, j_{a}}$.

- end iteration for $j$.

d) Find $m=\arg \max _{\{j=1, \ldots, N\}} q_{j}$.

e) Set $C_{m}=C_{m}-1$ and $C_{m_{a}}=C_{m_{a}}+1$.

f) Update the subchannel indexes of layer $l, l \in$ $\left[\min \left\{m, m_{a}\right\}, \max \left\{m, m_{a}\right\}\right]$. And recompute $P S_{l}$ which will involve the optimization of the bit efficiency. The other layers remain the same.

g) If no feasible $m$ is found, then the subchannel distribution efficiency is regained, go to 2), otherwise go to c).

The above algorithm provides adaptive loading capability. For each optimization, the initial subchannel distribution as well as the power and bit rate assignment are chosen to be the one designed for the latest channel condition and input characteristics. Assuming a slight change each time, the algorithm can converge to the new solution quickly.

The proposed loading algorithm can also be applied to solve the problem of maximizing data throughput for a given power constraint. This is indeed important since it derives the maximum data throughput by making the best use of available channel capacity where the modem should and does transmit at full power. In this case, we still choose the number of subchannel distribution as the optimization parameter. The optimization needs to compute not only the optimal transmitted power assigned to each user, but also the maximum number of bits from each layer to be transmitted per frame defined earlier as $\left\{B_{i}\right\}_{i=1}^{N}$. The algorithm starts from an arbitrary $B=\sum_{i=1}^{N} B_{i}$ and using the same algorithm for power minimization to compute the transmitted power consumption. It should be pointed out that $\left\{B_{i}\right\}_{i=1}^{N}$ can be computed based on $B$ and the data rate requirements of the layers. If the power consumption is below the constraint, then the throughput is increased by adding one bit into each layer $B_{i}=B_{i}+1$, which yields $B=B+N$. It can be solved by finding the best subchannel occupied by each layer to increase its data bit by one. The algorithm also checks if the subchannel distribution efficiency and total subchannel efficiency are violated by the action and then optimizes the subchannel allocation until the efficiencies are satisfied. If the computed power consumption is too high, the algorithm reduces one bit from each layer and optimizes the subchannel assignment to satisfy the efficiencies.

\section{Simulation Result}

This section will present the simulations carried out on typical layered source data. The performances of both the parallel transmission and the serial transmission are illustrated for comparison. The data throughput and source input are designed to be identical for the two transmission schemes in order to achieve a fair comparison. The simulation results under different channel parameters and source parameters are also compared and studied.

\section{A. Subband Coded Image}

We apply the proposed loading algorithm to subband coded image transmission over ADSL. Each subband is classified as a layer. The number of subchannels allowed in MCM is $C_{T}=256$. The bit rate is limited to the range $R_{\min }=2$ and $R_{\max }=6$. The images are four level subband decomposed using Daubechies 16 wavelet filter and then vector quantized using full search LBG algorithm. The quantized results are fix length coded in order to achieve more robustness against channel noise. For simplicity, we assume that subband 1 has BER1 requirement which corresponds to $\Gamma_{1}$, subband 2 and 3 
TABLE III

SIMULATION RESUlt FOR INTEGRATED SERVICE

\begin{tabular}{c|cc|cc}
\hline \multirow{2}{*}{ Data } & \multicolumn{4}{|c}{ Average Power(dB) } \\
\cline { 2 - 5 } Throughput & \multicolumn{2}{|c}{$R_{\max }=6$} & \multicolumn{2}{c}{$R_{\max }=12$} \\
\cline { 2 - 5 } & Parallel & Serial & Parallel & Serial \\
\hline \hline 448 & 19.88 & 20.10 & 18.60 & 19.17 \\
\hline 512 & 22.07 & 22.34 & 20.65 & 21.10 \\
\hline 576 & 24.26 & 24.70 & 22.20 & 22.86 \\
\hline 640 & 26.19 & 26.63 & 24.00 & 24.50 \\
\hline 1024 & 37.16 & 37.48 & 32.30 & 32.54 \\
\hline
\end{tabular}

TABLE IV

BER DISTRIBUTIONS FOR DIFFERENT REQUIREMENTS

\begin{tabular}{c|c|c|c}
\hline Requirement & Service 1 & Service 2 & Service 3 \\
& $200 \mathrm{~K}$ bits/s & $64 \mathrm{~K}$ bits/s & $10 \mathrm{~K} \mathrm{bits/s}$ \\
\hline L1 & $10^{-6}$ & $10^{-5}$ & $10^{-3}$ \\
\hline L2 & $10^{-5}$ & $10^{-3}$ & $10^{-6}$ \\
\hline L3 & $10^{-3}$ & $10^{-5}$ & $10^{-6}$ \\
\hline
\end{tabular}

with BER2 $\left(\Gamma_{2}\right)$ and the rest with BER3 $\left(\Gamma_{3}\right)$. The simulation results of the serial transmission are obtained by applying the loading algorithm described in Section IV-A and varying the frame power during each layer's transmission. Table II shows the averaged transmitted power per subchannel, defined as frame power $/ C_{T}$, at different BER distributions using "Lena" coded at $0.5 \mathrm{bpp}$ with data throughput $B_{T}=512$. The results demonstrate advantages of the parallel transmission over the serial transmission. The performance improvement increases as the difference among BER1, BER2, BER3 increases. Figs. 4 and 5 plot the performance comparison as a function of data throughput $B_{T}(448,512,576$, and 640 bits per frame), where the parallel transmission achieves as much as $2.5-\mathrm{dB}$ PSNR improvement or $2-\mathrm{dB}$ transmitted power gain. Increasing $B_{T}$ also increases the power consumption. The received images are shown in Fig. 6.

\section{B. Integrated Service of Video, Voice, and Data}

Wireline transmission systems will have to meet the needs of transmitting a variety of difference services, such as video, voice, and data. These services are associated with different data rates and different QoS requirement (in terms of BER), as described in Section II. We apply the parallel transmission scheme to the integrated services shown in Table I. Based on the assumption that each service is accompanied by an efficient rate control scheme to achieve constant bit rate, the number of bits to be

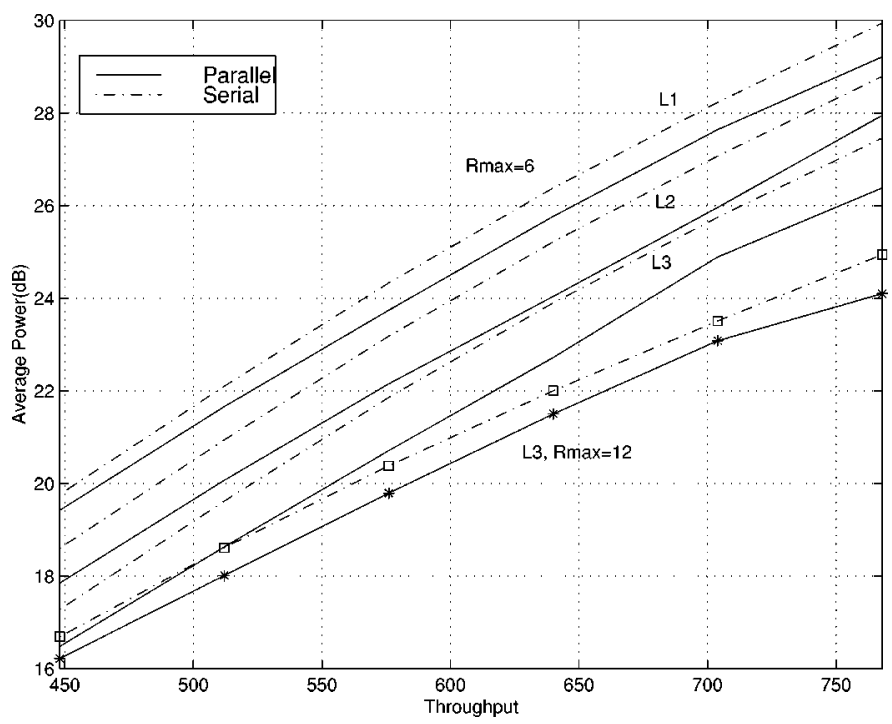

Fig. 7. Transmitted power consumption versus data throughput for $R_{\max }=6$.

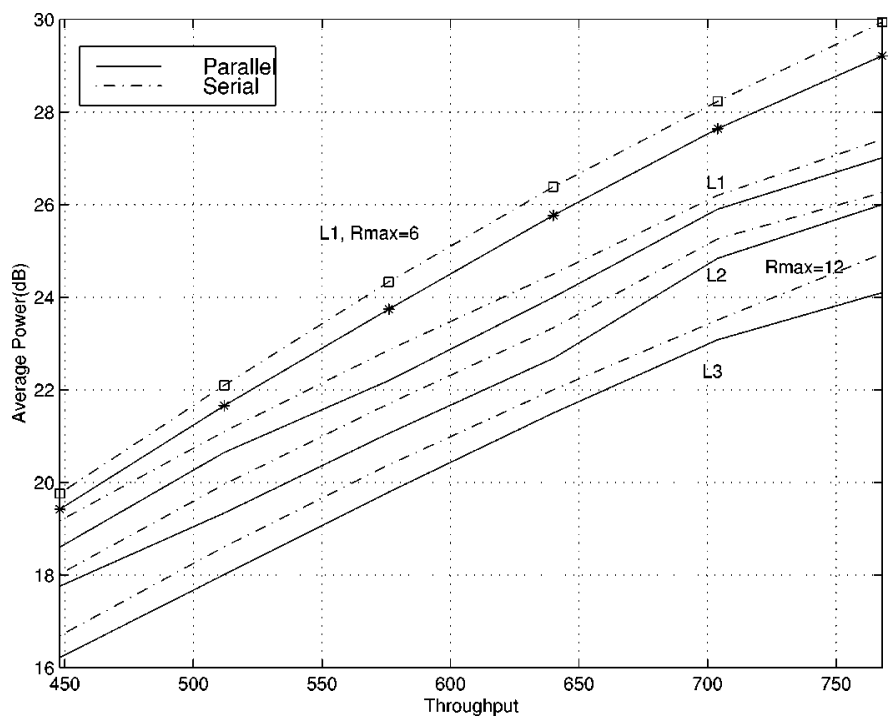

Fig. 8. Transmitted power consumption versus data throughput for $R_{\max }=$ 12

transmitted for each layer in an ADSL frame can be computed for a given data throughput and sampling rate. As we pointed out before, the proposed parallel transmission has the advantage that if the content over the link has to change periodically, the power and bit rate assignment remains the same, while for the serial transmission, frequent updating at both transmitter and receiver is required and thus yields inefficiency.

The numerical results shown in Table III are based on the integrated service whose components are illustrated in Table I. The transmitted power consumptions for the serial and parallel system are compared, as a function of the transmission parameter $B_{T}$ and $R_{\max }$. Additional modulator selection (higher $R_{\max }$ ) helps to reduce the power consumption. The parallel transmission achieves as much as $0.5-\mathrm{dB}$ power gain compared to the serial transmission.

Table IV shows the performance at different customer requirements. The effect of $R_{\max }$ is illustrated in Figs. 7 and 8 as 
$B_{T}$ increases from 448 to 764 . For L1, the extra 6 modulators leads to nearly $2-\mathrm{dB}$ power gain for the parallel transmission.

\section{CONCLUSION}

We unveiled a new transmission system for delivering integrated multimedia services over ADSL. By using a robust parallel transmission framework, the transmitted power consumption is reduced for a specified QoS distribution under a data throughput constraint. The services are classified into layers with different QoS requirement (in terms of BER), which are transmitted simultaneously through different subchannels. Such a scheme provides unequal error protection naturally by transmitting the services with higher priority through the subchannels with higher channel gain. The framework includes a loading algorithm which distributes the transmitted power and bit rate among the subchannels, in order to fully utilize the channel resources and enhance performance. Compared to the schemes designed for the services containing only data, the proposed parallel transmission achieves $0.5-2-\mathrm{dB}$ gain in terms of the average transmitted power per subchannel, by exploiting the characteristics of wireline channel. Overall, the proposed scheme opens the door to the robust and efficient delivery of integrated multimedia services over traditional telephone lines.

\section{REFERENCES}

[1] K. Maxwell, "Asymmetric digital subscriber line: Interim technology for the next forty years," IEEE Commun. Mag., pp. 100-106, Oct. 1996.

[2] J. A. C. Bingham, "Multicarrier modulation for data transmission: An idea whose time has come," IEEE Commun. Mag., May 1990.

[3] J. Campello de Souza, "Optimal discrete bit loading for multicarrier modulation systems," presented at the IEEE Symp. Information Theory, Boston, 1998.

[4] P. S. Chow, J. M. Cioffi, and J. A. C. Bingham, "A practical discrete multiton transceiver loading algorithm for data transmission over spectrally shaped channels," IEEE Trans. Commun., pp. 773-775, Feb./Mar./Apr. 1995.

[5] B. S. Krongold, K. Ramchandran, and D. L. Jones, "Computationally efficient optimal power allocation algorithm for multicarrier communication systems," in Proc. ICC98, Atlanta, GA, June 1998.

[6] R. F. H. Fisher and J. B. Huber, "A new loading algorithm for discrete multiton transmission," in Proc. GLOBECOM'96, pp. 724-728.

[7] T. J. Willink and P. H. Wittke, "Optimization and performance evaluation of multicarrier transmission," IEEE Trans. Inform. Theory, vol. 43, pp. 426-440, Mar. 1997.

[8] “MPEG-4 visual final committee draft,", Tech. Rep., May 1998.

[9] H. Zheng and K. J. R. Liu, "Robust image and video transmission over spectrally shaped channels using multicarrier moduation," IEEE Trans. Multimedia, no. 1, pp. 88-103, Mar. 1999.
[10] _ _ "Robust H.263 video transmission over spectrally shaped channels using multicarrier modulation," in IEEE Proc. Workshop Multimedia Signal Processing, Los Angeles, CA, Dec. 1998.

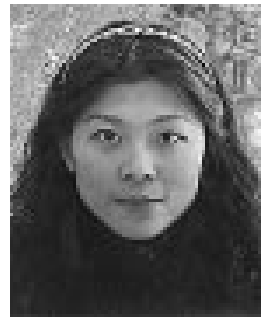

Haitao Zheng (S'97-M'00) received the B.S. degree in electrical engineering from Xian Jiaotong University, Xian, China, in 1995, and the M.S. and Ph.D. degrees in electrical engineering from the University of Maryland, College Park, MD in 1998 and 1999 respectively.

Currently she is with the Wireless Communications Research Department, Bell Labs, Lucent Technologies, Holmdel, NJ, where she is a Member of the Technical Staff. During 1995-1999, she was an Institute for Systems Research Fellow and Research Assistant in the Digital Signal Processing Laboratory at the University of Maryland, College Park. She received the 1998-1999 "George Harhalakis" Outstanding Systems Engineering Graduate Student Award in recognition of outstanding contributions in cross-disciplinary research, from the Institute for Systems Research, University of Maryland, College Park. Her current research interests include multimedia coding and communications, especially wireless multimedia communications, packet video, and joint source/channel coding.

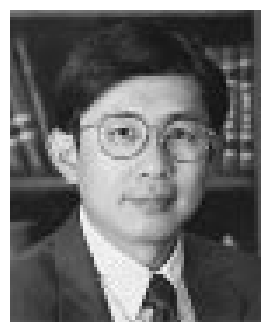

K. J. Ray Liu (S'86-M'90-SM'93) received the B.S degree from the National Taiwan University in 1983 , and the Ph.D. degree from the University of California, Los Angeles, in 1990, both in electrical engineering.

Since 1990, he has been with the Electrical Engineering Department and Institute for Systems Research of the University of Maryland at College Park, where he is a Professor. During his sabbatical leave in 1996-1997, he was a Visiting Associate Professor at Stanford University. His research interests span various aspects of signal/image processing and communications. He has published over 160 papers, of which over 60 are in archival journals and book chapters.

Dr. Liu received numerous awards including the 1994 National Science Foundation Young Investigator Award, the IEEE Signal Processing Society's 1993 Senior Award (Best Paper Award), the IEEE Beneluz Joint Chapter on Vehicular Technology and Communications 1999 Award, the George Corcoran Award in 1994 for outstanding contributions to electrical engineering education, and the 1995-1996 Outstanding Systems Engineering Faculty Award in recognition of outstanding contributions in interdisciplinary research, both from the University of Maryland, and many others. Dr. Liu was an Associate Editor of IEEE TRANSACTIONS ON Signal PROCESSING, a Guest Editor of special issues on Multimedia Signal Processing of PROCEEDINGS OF THE IEEE, and a Guest Editor of special issue on Signal Processing for Wireless Communications of IEEE JouRNAL OF SELECTED AREAS IN COMMUNICATIONS. He currently serves as the Chair of Multimedia Signal Processing Technical Committee of IEEE Signal Processing Society, a Guest Editor of the special issue on Multimedia over IP of the IEEE TRAnsaCtions ON Multimedia, a Guest Editor of the special issue on Robust Multimedia Transmission of IEEE Signal Processing MAGAZINE, an editor of the Journal of VLSI Signal Processing Systems, and the series editor of the Marcel Dekker series on signal processing. 